\title{
Higher-order Topological Phase in a Simple Two- dimensional Dielectric Photonic Crystals
}

\author{
Guanxia Yu ( $\nabla$ sys@njfu.edu.cn ) \\ Yu Xiaomeng \\ nanjing Forestry university \\ shen $\mathrm{Yu}$ \\ Nanjing Forestry University \\ Wang Haodong \\ Nanjing Forestry University \\ Yuan Guangyao \\ Nanjing Forestry University
}

Nanjing Forestry University https://orcid.org/0000-0001-5007-1059

\section{Research Article}

Keywords: PT symmetric, Extraordinary transmission, Multi-pairs nonreciprocal modes

Posted Date: February 14th, 2022

DOI: https://doi.org/10.21203/rs.3.rs-1188271/v1

License: (c) (i) This work is licensed under a Creative Commons Attribution 4.0 International License.

Read Full License 


\title{
Multiple Nonreciprocal transmission in the PT-symmetric system with defect layer
}

\author{
Guanxia $\mathrm{Yu}^{1,3 *}$ Xiaomeng $\mathrm{Yu}^{2}$, Yu Shen ${ }^{3}$, Haodong Wang ${ }^{3}$, Guangyao Yuan ${ }^{3}$ \\ 1 College of Science, Nanjing Forestry University, Nanjing 210037, P. R. China. \\ 2 College of Materials Science and Engineering, Nanjing Forestry University, Nanjing 210037, P. R. China. \\ 3 College of Information Sciences and Technology, Nanjing Forestry University, Nanjing 210037, P. R. China. \\ *E-mail:sys@njfu.com.cn
}

\begin{abstract}
In this paper, a one-dimensional magneto-optic(MO) multilayer structure with the nonreciprocal transmission of dual-mode is designed. The energy band and transmission properties of composite structures are studied based on transfer matrix theory. Two pairs of nonreciprocal dispersive curves corresponding to edge modes appear in the bandgap. When the gain and loss factors are introduced into the multilayer structure, tunable and nonreciprocal extraordinary transmission appears above PT thresholds. Significantly, the composite structure with small and unequal gain/loss factors induces extraordinary transmission and keeps nonreciprocal frequencies stable. Such distinguishing properties may provide these structures with attractive applications as optical isolators and multichannel nonreciprocal optical devices.
\end{abstract}

Keywords: PT symmetric, Extraordinary transmission, Multi-pairs nonreciprocal modes.

\section{1 introduction}

A non-Hermitian system with parity-time symmetry(PT) proposed by Bender[1,2] has attracted extensive attention, extending the framework of quantum mechanics from the real domain into the complex domain. Among the non-Hermitian system, it is counter-intuitive that non-Hermitian Hamiltonians also exhibit entirely real eigenvalue spectra below a symmetry-breaking point (exceptional points, EPs). Another fascinating property is the existence of spontaneous PT-symmetry above the EPs where the systems can undergo an abrupt phase transition, and the eigenvalues of the non-Hermitian Hamiltonian of the systems abruptly turn from real to complex. Although such a class of non-Hermitian Hamiltonians has originated from the system of quantum mechanics, the developments are still limited within the framework of theoretical physics due to the difficulty in realizing PT-symmetry in electronic systems. Motivated by fundamental studies of PT-symmetric quantum Hamiltonians, the idea has been fruitfully extended to other areas by PT-symmetric Hamiltonians. Especially in the optical and acoustic PT system, a series of ideal experimental platforms further verify the concept of parity-time symmetry.

Because the beam propagation equation under paraxial approximation in optical systems is mathematically consistent with the nonlinear Schrodinger equation in quantum mechanics[3,4], a nonHermitian PT-symmetric system can be realized and observed in optical structures, such as waveguide and periodic structures. A periodic optical structure with PT invariance is realized by the 

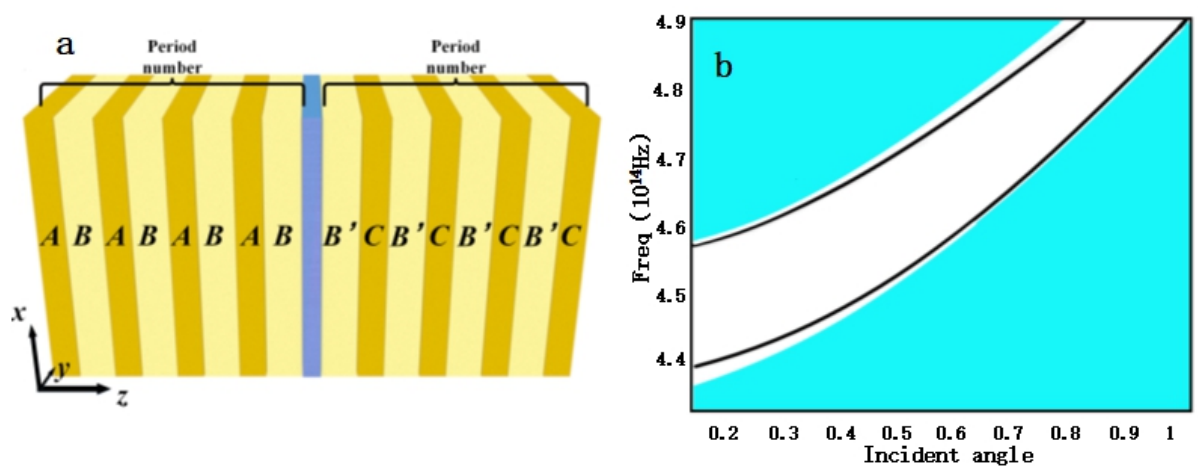

Figure 1: (a) Schematic of the composed multilayered structure with defect layer $(A B)^{N} D\left(B^{\prime} C\right)^{N}$, where layers $B$ and $B^{\prime}$ are filled with MO materials, layers $A$ and $C$ are filled with gain and loss materials, respectively, and layer $D$ is normal medium.(b)Two one-way dispersive curves in the band structure of the composite system, where $B$ and $B^{\prime}$ is no external magnetic fields, and gain/loss factors in the layers $A$ and $C$ are 0 .

introduction of a dielectric medium with a complex refractive index profile satisfying $n^{*}(r)=n(-r)$, which is equivalent to the potential function $\mathrm{V}(\mathrm{r})$ in the non-Hermitian Hamiltonian system of quantum mechanics. In the past few years, several new ideas in complex optical structures have continuously been inspired researchers. Various unconventional light propagation features have been studied when PT-symmetry breaking occurs at PT thresholds. A range of associated extraordinary phenomena have also been proposed in optical PT-symmetric system, including unidirectional invisibility [5,6], optical isolation[7], nonreciprocal Bloch oscillations[8,9], laser absorber[10,11], unidirectional invisibility[12-14] and various extraordinary nonlinear effects [15-17].

In this work, a PT-symmetric system consisting of two magneto-optical multilayer structures with a defect layer is constructed(Fig.1). The nonreciprocal transmission characteristics of interface modes, also referred to as optical Tamm states (OTSs) due to overlapping band gaps, have been investigated at the interface of two multilayer structures. Different from the previous study[1820], there are two pairs of nonreciprocal transmission peaks in one bandgap as the defect layer's thickness is selected appropriately. With increasing gain/loss factors, the extraordinary transmission of two pairs of nonreciprocal frequencies appears. The paper is arranged as follows: firstly, transmission and reflection coefficients of the composite structure is rigorously analyzed by the transfer matrix methods[18,21], then energy band characteristics and nonreciprocal transmission spectrum of the composite structure are investigated. Moreover, the extraordinary nonreciprocal transmission in the PT-symmetric system is discussed with different external static magnetic fields and gain/loss factors.

\section{Theoretical model and methods}

Our PT-symmetric system is a composite periodic structure with a defect layer $D$ sandwiched by two periodic structure $(A B)^{N} D\left(B^{\prime} C\right)^{N}$, where $N$ is the periodic number, as shown in Fig.1a. In a unit, layers $B$ with thickness $d_{B}$ and $B^{\prime}$ with thickness $d_{B^{\prime}}$ are magneto-optical (MO) dielectric(bismuth iron garnet:BIG) applied on the opposite static magnetic fields, and the permittivity of layer $B\left(B^{\prime}\right)$ 

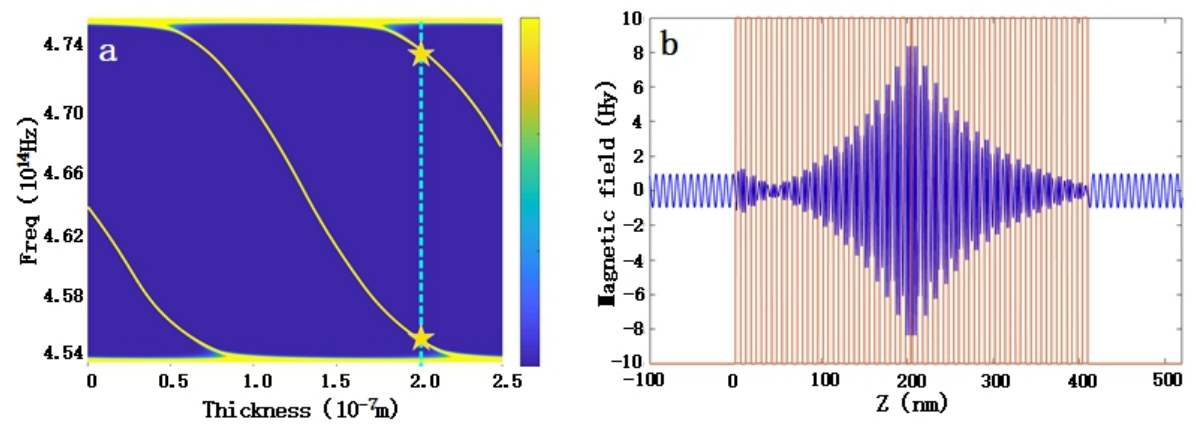

Figure 2: (a)Relationship between the dispersive curves in the band gap and thickness of defect layer with an incident angle $\pi / 5$, where two asterisks in the dispersive curves indicate that the same thickness corresponds to two one-way frequencies $\left(4.55 \times 10^{14} \mathrm{~Hz}\right.$ and $\left.4.73 \times 10^{14} \mathrm{~Hz}\right)$.(b)Distribution of out-of-plane magnetic field at $f=4.55 \times 10^{14} \mathrm{~Hz}$ along $z$-direction with $x=0$, where an incident angle is $\pi / 5$.

in the Cotton-Mouton(Voigt) geometry is characterized by a tensor[22]:

$$
\varepsilon_{B}=\left[\begin{array}{ccc}
\varepsilon_{1} & 0 & i \Delta_{g} \\
0 & \varepsilon_{1} & 0 \\
-i \Delta_{g} & 0 & \varepsilon_{1}
\end{array}\right]
$$

where $\Delta_{g}$ originating from the $\mathrm{MO}$ effect is an off-diagonal element, and its positive and negative values are determined by the external magnetic field along y-directions. Assuming that layer $A$ with thickness $d_{A}$ and layer $C$ with thickness $d_{C}$ are gain and absorptive quantum dots doped $\mathrm{SiO}_{2}$, and the optical dielectric constant distributions are denoted as $\varepsilon_{\mathrm{A}}=\varepsilon_{\mathrm{h}}-i \rho$ and $\varepsilon_{C}=\varepsilon_{\mathrm{h}}+i \rho$, respectively, where $\rho$ is the gain/loss factor. $\varepsilon_{\mathrm{h}}$ represents the permittivity of the host material of layers A and C, which can be described by Sellmeier dispersion relation[23]: $\epsilon_{r A}=\epsilon_{r C}=1+\sum_{i} c_{i} \lambda^{2} /\left(\lambda^{2}-\lambda_{i}^{2}\right), i=1,2,3$, where $\lambda$ denotes the incident wavelength, $c_{i}$ represents the oscillator strength constant, which values are $0.7,0.41$, and 0.9 , and $\lambda_{i}$ is the characteristic oscillator wavelength, which values are $68 \mathrm{~nm}, 116 \mathrm{~nm}$, and $9896 \mathrm{~nm}$, respectively. The relative permeability of all materials in the complex structure is 1 . In a rectangular coordinate system, the defect layer $D$ with thickness $d_{D}$ located in the center of the coordinate is normal dielectric, and two periodic structure $(A B)^{N}$ with the period $\Lambda_{L}\left(d_{A}+d_{B}\right)$ and $\left(B^{\prime} C\right)^{N}$ with the period $\Lambda_{R}\left(d_{B^{\prime}}+d_{C}\right)$ are arranged along $z$-direction. If the thicknesses of the layers of $A, B, B^{\prime}$, and $C$ are the same, the left and right parts of the composite structure satisfy PT symmetry, which is different from the PT symmetry of the units in previous studies[20, 24, 25].

Without loss of generality, a TM plane wave $H_{y}(x, z)=H_{y 0} e^{i k_{x} x+i k_{z} z}$ with a frequency $\omega$ along $y$-direction is incident at incident angle $\theta$ from boundaries, where $k_{x}$ and $k_{z}$ are the wave vectors along $x$ - and $z$-directions, respectively. According to Maxwell's equations and electromagnetic field boundary conditions, the wave propagation behaviors in multilayer structures can be theoretically calculated using the transfer matrix method (TMM) $[18,21]$. For the left periodic system $(A B)^{N}$, the transfer matrix is defined as: $T_{L}=M_{A B} P_{A} M_{B A} P_{B}$, where $P_{i}=\operatorname{diag}\left(e^{i k_{i z} d_{i}}, e^{-i k_{i z} d_{i}}\right)$ represents the phase-shift factors in one layer from the front boundary to the rear boundary, and $k_{i z}$ is defined as $k_{i z}=\sqrt{\left(\omega^{2} \epsilon_{i}^{2}-\Delta_{g i}^{2}\right) /\left(c^{2} \epsilon_{i}^{2}\right)-k_{x}^{2}}$. Here the matrix $M_{i j}$ in the interface between 
two adjacent layer can be expressed as:

$$
\mathrm{M}_{i j}=\frac{1}{2 M_{j}}\left[\begin{array}{cc}
M_{i}^{*}+M_{j} & M_{i}^{*}-M_{j}^{*} \\
M_{i}-M_{j} & M_{i}+M_{j}^{*}
\end{array}\right]
$$

where $M_{i(j)}=\left(k_{i(j) z}+i k_{x} \Delta_{g i(j)}\right) /\left(\omega \epsilon_{0} \epsilon_{i(j)}^{\prime}\right)$, and $\epsilon_{i(j)}^{\prime}=\Delta_{g i(j)}^{2}-\epsilon_{r i(j)}^{2}$. In the model, if the layers are isotropic dielectrics, and the off-diagonal elements $\Delta_{g i(j)}$ in their permittivity are reduced to zero. For the right periodic system $(B C)^{N}$, the transfer matrix $T_{R}$ can be derived similar to $T_{L}$. According to Bloch theorem, two Bloch wavevectors for the transfer $T_{R}$ and $T_{L}$ can be expressed in the form:

$$
e^{i K_{i} \Lambda_{i}}=\frac{1}{2}\left[\left(T_{11 i}+T_{22 i}\right) \pm \sqrt{\left(T_{22 i}-T_{11 i}\right)^{2}+4 T_{12 i} T_{21 i}}\right]
$$

where $K_{i}$ is Bloch wavevector, and $i=L, R$ denotes left and right periodic structures, respectively. To obtain the OTS at the interface between two periodic structures, the constant $K_{L}$ and $K_{R}$ must be complex, and have the opposite sign of their imaginary part to guarantee the field exponential decay in both sides. By evaluating the impedances of the Bloch waves in the left and right period structure, the dispersion relation for OTS modes is obtained[26,27],

$$
k_{z B} \frac{\epsilon_{D} T_{12 L} e^{-i k_{z d} d_{D}}+T_{12 L}-e^{i K \Lambda_{L}}}{\epsilon_{B} T_{12 L} e^{-i k_{z d} d_{D}}-T_{12 L}+e^{i K \Lambda_{L}}}=k_{z B^{\prime}} \frac{\epsilon_{D} T_{12 R} e^{-i k_{z d} d_{D}}+T_{12 R}-e^{i K \Lambda_{R}}}{\epsilon_{B^{\prime}} T_{12 R} e^{-i k_{z d} d_{D}}-T_{12 R}+e^{i K \Lambda_{R}}}
$$

Since the left and right multilayers are almost symmetrical, there are similar band diagrams. By solving the equation (4) numerically, the dispersion relation of two specific period structures is shown in Fig.1(b), where parameters of the multilayer are taken to be: $d_{A}=d_{C}=d_{B}=d_{B^{\prime}}=$ $600 \mathrm{~nm}, d_{d}=200 \mathrm{~nm}$, and the relative permittivity of layer $B, B^{\prime}$ and $D \epsilon_{B}=\epsilon_{B^{\prime}}=\epsilon_{D}=6.25$, $\Delta_{g B}=\Delta_{g B^{\prime}}=\Delta_{g D}=0$ with no external magnetic fields, and layer number $N=30$. Due to almost similar period structures, there are identical band diagrams for two-period structures(Fig.1b), where blue regions correspond to pass-bands, and white areas correspond to stop-band. Different from the complex structure consisting of a two-period structure with no defect layer[25], it is important to note that two dispersive curves of OTS near the edge of the energy band appear in the bandgap within frequency range $(4.3-4.9) \times 10^{14} \mathrm{~Hz}$. That is, there are two OTSs with different frequencies at a certain incident angle.

Because the OTSs are strongest at interfaces of the defect in the middle of two periods and decay exponentially outward along the two periodic structures[28], the defect layer's thickness impacts the evolution of the OTSs. In Fig.2a, the relationship between the dispersive curves and the defect layer's thickness is depicted with an incident angle $\pi / 5$. Obviously, the dispersive curve evolutes from upper bulk state to lower bulk state in the bandgap, and varies periodically with the defect layer's thickness. Within a certain thickness of the defect layer, there may be two OTS's frequencies, as shown in Fig.2a indicated by an asterisk in a vertical dashed line with thickness $d_{D}=200 \mathrm{~nm}$, which is consistent with the case in Fig.1b. To display the pattern of the OTS mode clearly, we plot the distribution of the field along the z-direction in Fig.2b. As expected, the field is strongly peaked at the interfaces of the defect layer between two-period structures and exponential decay on both sides away from the defect layer. Although there are two different frequencies at a specific incident angle in the bandgap, they belong to the OTSs with the same field patterns.

When the layer $B$ and $B^{\prime}$ are filled with MOMs applied the external magnetic fields with the 

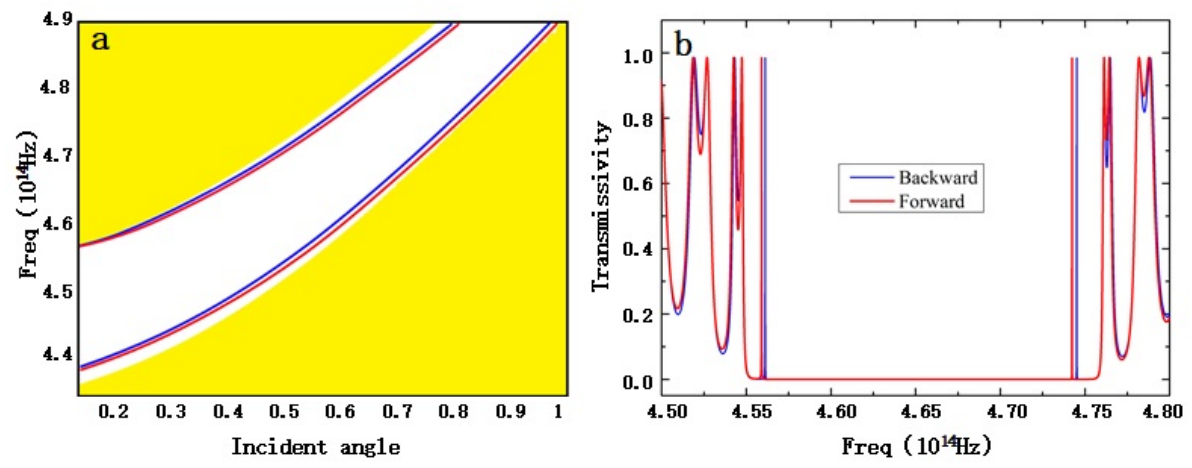

Figure 3: (a)Two pairs of nonreciprocal one-way dispersive curves in the band structure of the composite system, where $B$ and $B^{\prime}$ is filled with MO materials with different directions of the external magnetic fields, and gain/loss factors in the layers $A$ and $C$ are 0.(b)Transmission spectrum with an incident angle $\pi / 5$, where $\Delta_{g B}=-\Delta_{g B^{\prime}}=0.2$, and $\rho=0$.

opposite directions, the nonreciprocal transmission in the complex structure can be produced by the asymmetric gyrotropic elements(off-diagonal elements) in the MOMs. In the optical frequencies, the dispersion relation of electromagnetic parameters of MOMs with the external magnetic field is weak, and the diagonal elements of the relative permittivity are close to the $\operatorname{constant}\left(\epsilon_{B}=\epsilon_{B^{\prime}}=\right.$ $\left.\epsilon_{D}=6.25\right)$. Although the gyrotropic components are the key to nonreciprocal transmission, it must be noted that the gyrotropic components are small in the optical band[20]. To enhance the effect of the anisotropic magneto-optical(MO) effects, the value of the off-diagonal term is 0.2 . The band diagrams of two-period structures almost overlap due to the small gyrotropic components as other conditions are the same as in Fig. 1, as shown in Fig.3a. According to the formula(3), It could be vividly seen that there are two pair nonreciprocal dispersive curves of OTSs in the bandgap, where red and blue lines represent for forward and backward incidence, respectively. To clearly show the nonreciprocal transmission of two pair dispersive curves in the bandgap, the transmission spectrum within the frequencies range $(4.5-4.8) \times 10^{14} \mathrm{~Hz}$ is depicted based on TMM in Fig.3b, where an incident angle is $\pi / 5$. Two pairs of perfect nonreciprocal transmission peaks appear in the bandgap, and the frequencies of the forward incidence are larger than those of the backward. The two pairs of the nonreciprocal frequencies in the band gap could be considered as technologically important to flexibly design and fabricate the multichannel optical isolators or diodes and unidirectional photonic devices.

When the gain/loss factors are introduced in the layer $A$ and $C$, the complex structure is also a PT-symmetrical system under the combination of the parity $(\mathrm{P})$ operator and the time $(\mathrm{T})$ operator. It is well known that the composite system has a PT-symmetry phase under small gain/loss factors, and the original transmission properties are still maintained[1,2]. However, with the increase of gain/loss factors, the system encounters the broken symmetry phase, and appears extraordinary transmission. Figure 4 shows the relationships between the transmissivity of two pairs of nonreciprocal frequencies and gain/loss factors with the incident angle $\pi / 5$. It is observed that extraordinary transmission appears when the gain/loss factors are larger than 0.002 . It is also noted that the structure is sensitive to change of the small gain/loss factors, and the transmissivity increases rapidly with the gain/loss factors. Moreover, the extraordinary transmission of two pair nonreciprocal frequencies exhibits different properties with gain/loss factors. For the case 

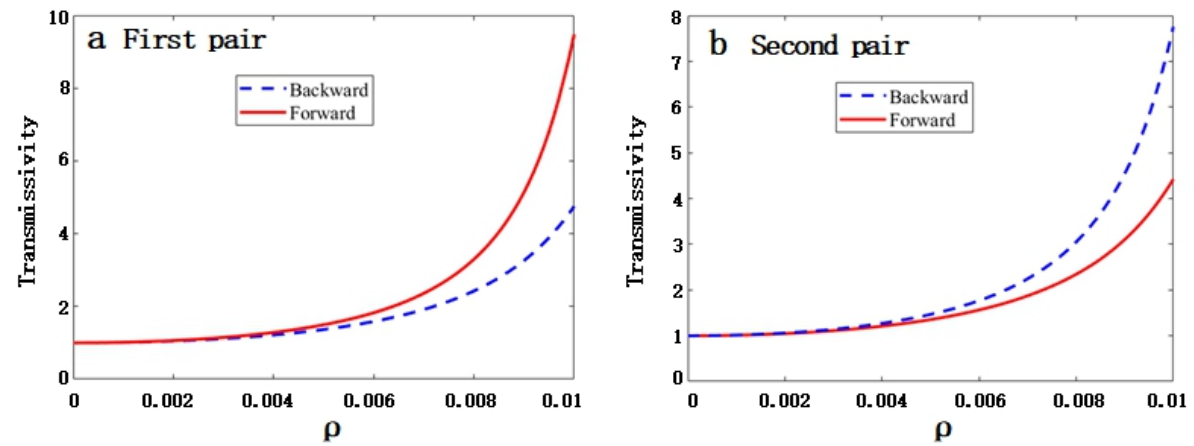

Figure 4: Relation between the transmissivity and gain/loss factors with incident angle $\pi / 5$, where the solid and dashed lines correspond to forward and backward incidence, respectively. (a)The first pair of nonreciprocal frequencies. (b)The second pair of nonreciprocal frequencies.
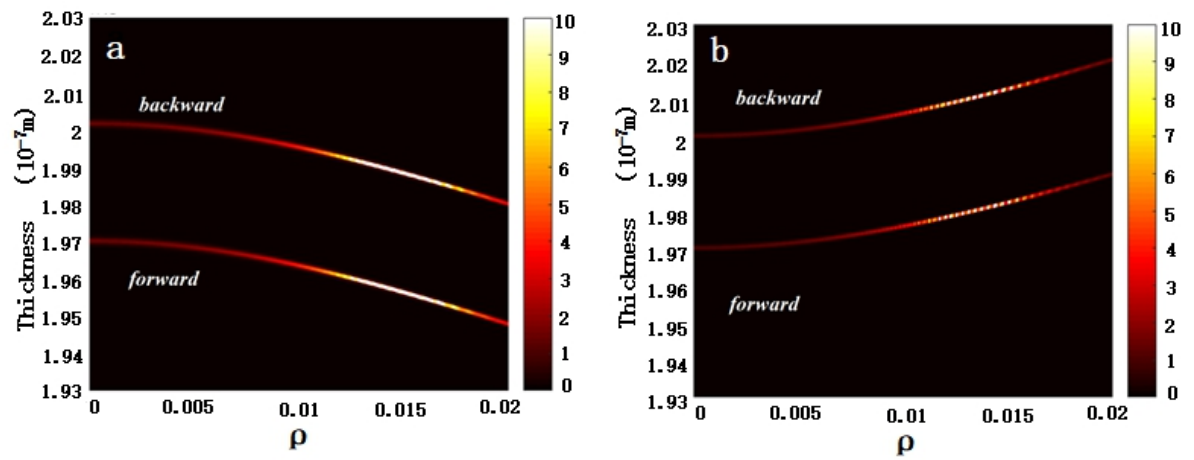

Figure 5: 2D transmissivity diagram of two pairs of non-reciprocal frequencies with different gain/loss factors and defect layer thickness. (a)The first pair of nonreciprocal frequencies. (b)The second pair of nonreciprocal frequencies. 


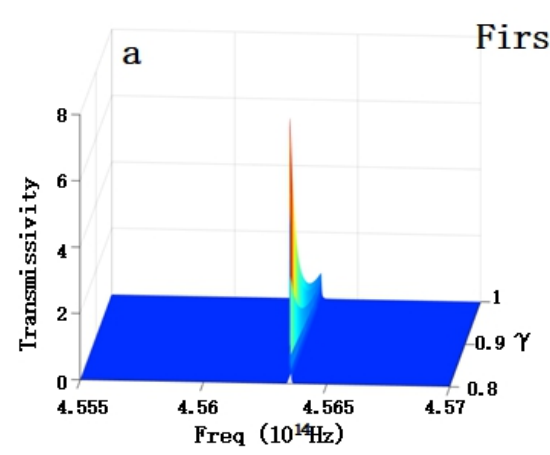

First pair b
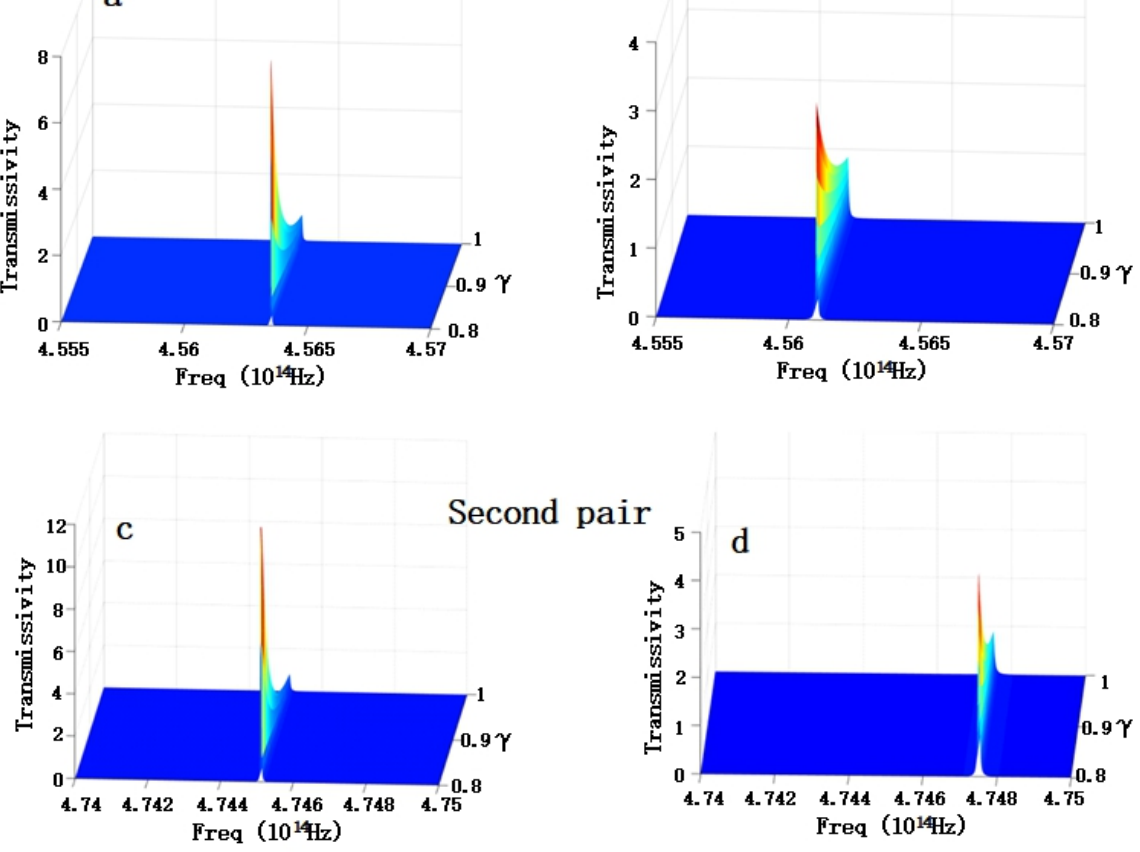

Figure 6: Effect of the asymmetric factor $\gamma$ on transmissivity of two pairs of nonreciprocal frequencies with incident angle $\pi / 5$. (a),(b) The first pair nonreciprocal frequencies of forward and backward incidence, respectively. (c)and (d) The second pair nonreciprocal frequencies of forward and backward incidence, respectively.

of the first pairs, the transmissivity of backward frequency increases faster than that of forward frequency(Fig. 4a), and the second pair is opposite to that of the first pair(Fig. 4b).

Based on the above, the generation of two pairs of nonreciprocal frequencies depends on the defect layer's thickness of(Fig.2a). It is of practical significance to obtain higher transmissivity with smaller gain/loss factors under certain thicknesses in the PT-symmetric system. When the incident angle $\pi / 5$ and the nonreciprocal frequencies remain unchanged, the effect of defect layer thickness and gain/loss factors on transmissivity is depicted in Fig.5, where the black area represents 0 transmissivities, and the bright-line shows different transmissivity. The figure shows that the defect layer thickness decreases with the increase of gain/loss factors for the first pair nonreciprocal frequen$\operatorname{cies}$ (Fig.5a), and the case is just the reverse for the second pair nonreciprocal frequencies(Fig.5b). It also noted that the space between the nonreciprocal frequencies of the two pairs does not change. That is, the nonreciprocal properties remain unchanged. However, the transmissivity does not simply increase with the gain/loss factor. When the defect thickness and gain/loss factor are set to a certain value, the transmittance reaches the maximum value(The brightest part of the bright-line). The results show us that the nonreciprocal devices with extraordinary transmittance are achieved by selecting the appropriate thickness of the defect layer and gain/loss factors.

It is well known that the extraordinary transmission depends on the PT symmetrical structure with the same gain and loss factors when PT-symmetry breaking occurs at PT thresholds. However, the gain and loss factors in layers $A$ and $C$ of the composite structure may not be strictly symmetrical in practice, which results in the composite system not satisfying PT symmetry. Therefore, it 
is necessary to discuss the effect of the PT-asymmetric structure on extraordinary transmission. To show this PT-asymmetry in the composite structure, an asymmetric factor $\gamma$ is defined as the ratio of loss factor to gain factor $\left(\gamma=\rho_{\text {gain }} / \rho_{\text {loss }}\right)$, where $\gamma$ deviation from 1 indicates that the structure is not PT-symmetric. Assuming incident angle $\pi / 5$ and a fixed loss factor $\rho=5 \times 10^{-4}$ in the layers $C$, the gain factors are changed from $\rho=5 \times 10^{-4}$ to $\rho=4 \times 10^{-4}$, that is, the asymmetric factors $\gamma$ is changed from 1 to 0.8 . The relationships between transmissivity of two pairs of nonreciprocal frequencies and the asymmetric factor $\gamma$ are plotted in Fig.6, where the $X-, Y$ - and $Z$-axis represent the frequencies, the asymmetric factor $\gamma$, and transmissivity, respectively. The figure shows no extraordinary transmission for two pairs of nonreciprocal frequencies as $\gamma$ is near one due to the little gain/loss factors. However, as $\gamma$ deviates from one, the transmissivity increases with the decrease of $\gamma$, and extraordinary transmission appears. The lager the asymmetric factor $\gamma$ of the structure, the greater the transmissivity. Although the gain/loss factor is too small to reach the PT thresholds in our composite system, a minor asymmetric factor $\gamma$ realizes PT broken symmetry and appears extraordinary transmission. In addition, it also shows that two pairs of nonreciprocal frequencies remains stable as the asymmetric factors $\gamma$ increase, which shows that the composite $\mathrm{PT}$ asymmetrical structure is favorable to extraordinary transmission and make it easy to fabricate multi-channel nonreciprocal devices.

\section{Conclusion}

In this paper, a composite PT symmetrical system with two periodic structures consisting of MO and gain/loss materials is designed, and the nonreciprocal properties of the edge state in the composite structure are investigated using the transfer matrix method. As a defect layer introduced in the centre of the composite structure with a specific thickness, two pairs of nonreciprocal dispersive curves appear in the bandgap due to the opposite magnetic fields of MO materials in two periodic structures. Furthermore, the extraordinary transmission of two pairs of nonreciprocal frequencies can be achieved when the gain/loss factors increase to the PT threshold. Most important, the PT asymmetric structure with small and unequal gain/loss factors also induce extraordinary transmission and keep nonreciprocal frequencies stable. The designed composite structure makes the design and manufacture of the multi-channel nonreciprocal devices more practical.

\section{References}

[1] C. M. Bender and S. Boettcher, Real spectra in non-Hermitian Hamiltonians having PT symmetry, Phys. Rev. Lett., 80: 5243-5246 (1998).

[2] C. M. Bender, S. Boettcher, and P. N. Meisinger, PT-symmetric quantum mechanics, J. Math.Phys. 40: 2201-2229(1999).

[3] K. G. Makris, R. El-Ganainy, and D. N. Christodoulides, Beam dynamics in PT symmetric optical lattices, Phys. Rev. Lett.100, 103904 (2008).

[4] S. Longhi, Optical Realization of Relativistic Non-Hermitian Quantum Mechanics, Phys. Rev. Lett. 103, 123601 (2009). 
[5] A. Regensburger, C. Bersch, M.-A. Miri, G. Onishchukov, D. N. Christodoulides, and U. Peschel, Parity-time synthetic photonic lattice Nature 488, 167-171 (2012)

[6] Z. Lin, H. Ramezani, T. Eichelkraut, T. Kottos, H. Cao, and D. N. Christodoulides, Unidirectional invisibility induced by P T-symmetric periodic structures Phys. Rev. Lett. 106, 213901 (2011).

[7] L. Chang, X. Jiang, S. Hua, C. Yang, J. Wen, L. Jiang, G. Li, G. Wang, and M. Xiao, ParityTime Symmetry and Variable Optical Isolation in Active-Passive-Coupled Microresonators, Nat. Photonics 8, 524 (2014).

[8] S. Longhi, Bloch Oscillations in Complex Crystals with PT Symmetry, Phys. Rev. Lett. 103(12):123601(2009).

[9] S. Ding, P. W. Guo, Nonreciprocal optical Bloch-Zener oscillations in ternary parity-timesymmetric waveguide lattices, App. Phy. Lett., 100(15):151913(2012)

[10] Y. D. Chong, L. Ge, and A. D. Stone, P t-symmetry breaking and laser-absorber modes in optical scattering systems, Phys. Rev. Lett. 106, 093902(2011).

[11] Y. Sun, W. Tan, H. Li, J. Li, and H. Chen, Experimental demonstration of a coherent perfect absorber with PT phase transition, Phys. Rev. Lett. 112, 143903(2014).

[12] A. Regensburger, C. Bersch, M. A. Miri, G. Onishchukov, D. N. Christodoulides,and U. Peschel, Parity-time synthetic photonic lattices, Nature,488:167-171(2012).

[13] S. Longhi, Parity-Time Symmetry meets Photonics: A New Twist in non-Hermitian Optics, Europhys. Lett. 120: 64001(2017).

[14] L. Jin, X. Z. Zhang, G. Zhang, Z. Song, Reciprocal and unidirectional scattering of parity-time symmetric structures, Sci. Entific Rep. 6:32919(2016).

[15] G. X. Yu, X. M. Zhang, R. Y. Cao, and Y.H. Lv, Unidirectional transmission in twodimensional photonic crystal composed of parity-time symmetric and magneto-optical materials, Opt. Eng., 59(11):117102(2020)

[16] S. Longhi, PT-symmetric laser absorber, Phys. Rev. A., 82(3): 031801(2010).

[17] Y. D. Chong, L Ge, and A. D. Stone, PT-Symmetry Breaking and Laser-Absorber Modes in Optical Scattering Systems, Phys. Rev. Lett., 106: 093902 (2011)

[18] Y. T. Fang, Y. Y. Wang, and Y. C. Zhang, Twofold unidirectional properties of parity-time symmetry structure under magneto-optical modulation, Opt. Eng., 57(2): 027102 (2018)

[19] G. X. Yu, Y. H. Lv, X. M. Zhang and R.Y. Cao, Electromagnetic propagation characteristics of one-dimensional photonic crystals with metal layers in quasi-parity-time (PT)-symmetric system, Z. Naturforsch., 75(7): 665-670(2020).

[20] G. X. Yu, H. Z. Yang, J. J. Fu, X. M. Zhang, R. Y. Cao, Nonreciprocal transmission using a multilayer magneto-optical dispersive material with defect, J. Electromagnet. Wave., 34(10):1400-1409(2020). 
[21] Y. T. Fang, Tunable Nonreciprocal Tunneling Based on Nonsymmetric Magnetoplasmonic Resonance Structure, Plasmonics, 9, 1133-1141(2014).

[22] J. A. Kong, Electromagnetic Wave Theory, A Wiley-Interscience Publication, (Hoboken, NJ, USA 1990).

[23] S. Ding, G. P. Wang, Extraordinary reflection and transmission with direction dependent wavelength selectivity based on parity-time-symmetric multilayers, J. Appl. Phys., 117:023104(2015).

[24] Y. T. Fang, Y. X. Ni, H. Q. He, J. X. Hu, Effect of hybrid state of surface plasmon-polaritons, magnetic defect mode and optical Tamm state on nonreciprocal propagation, Opt. Comm., 320: 99-104(2014).

[25] H. Y. Dong, J. Wang, and K. H. Fung, One-way optical tunneling induced by nonreciprocal dispersion of Tamm states in magnetophotonic crystals, Opt. Lett., 38(24):5232-5235(2013).

[26] P. Yeh, Optical Waves in Layered Media, 2nd ed. (John Wiley and Sons, USA, 2005).

[27] I. Iorsh, A. Orlov, P. Belov, and Y. Kivshar, Spontaneous emission enhancement in metaldielectric metamaterials, Appl. Phys. Lett. 99, 151914 (2011).

[28] A. V. Kavokin, I. A. Shelykh, and G. Malpuech, Lossless interface modes at the boundary between two periodic dielectric structures, Phy. Rev. B., 72, 233102(2005). 